\title{
Uber die Beziehung zwischen der blutgerinnungsfördernden Wirkung der Gallussäure und ihrer chemischen Konstitution.
}

\author{
Von \\ Yutaka Noda und Takeo Kurakake.

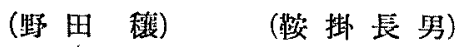 \\ (Aus dem Pharmakologischen Institut der Kaiserlichen \\ Tohoku Universität zu Sendai. Direktor : \\ Prof. S. Yagi.)
}

In einer vorangegangenen Mitteilung hat einer ${ }^{1)}$ von uns gezeigt, dass sowohl die stomakale als auch die intravenöse Darreichung von Natriumsalzen der Gerb- sowie der Gallussäure bei Kaninchen die Gerinnbarkeit des Blutes, wenn auch nicht erheblich, so doch ziemlich deutlich steigert, woraus er den Schluss gezogen hat, dass bei innerlicher Darreichung von gerbsäurehaltigen Drogen die darin vorhandene Gerbsäure im Darm in die Gallussäure umgewandelt und diese als Alkalisalz resorbiert wird, um die Gerinnung des Blutes zu beschleunigen. Um nun feststellen zu können, von welcher Atomgruppe im Molekül der Gallussäure deren gerinnungsfördernde Wirkung abhängig ist, stellten wir die vorliegende Untersuchung an, bei denen der Einfluss der Gallussäure und ihrer chemisch verwandten Substanzen auf die Gerinnbarkeit des Blutes von Tieren beobachtet wurde.

Die Untersuchungen wurden, wie die vorangegangenen, in einer thermostatischen Kammer von $20^{\circ} \mathrm{C}$ an Kaninchen vorgenommen, deren Blut 4 Stunden lang alle 30 Minuten aus der hinteren Ohrmuschelvene entnommen wurde, um dessen Gerinnungszeit mittels des Koagulometers nach Brodie, Russel und Bogg ${ }^{2)}$ zu bestimmen. Nach der zweiten Blutentnahme wurden die zu prüfenden Substanzen in wässerigen Lösungen in die andere Ohrvene der Tiere einverleibt, um die Gerinnbarkeit vor und nach der Einverleibung zu vergleichen.

1) Nod a, Tohoku Journ. Exp. Med., 1938, 33, 247.

2) Brodie, Russel u. Bogg, z. n. Abderhalden, Handbuch der biologischen Arbeitsmethoden, Berlin $\mathrm{u}$. Wien, 1924, IV, 3, 217. 
Mit jeder zu prüfenden Substanz wurden 5 Versuche angestellt, und von den Gerinnungszeiten der Blutproben, die im entsprechenden Zeitpunkt entnommen wurden, der Durchschnitt genommen. Jede durchschnittliche Gerinnungszeit wurde dann in der Voraussetzung: umgerechnet, dass die der zweiten Blutprobe 100 ist, und in Abbildungen zusammengestellt, um sie im relativen Wert miteinander vergleichen zu können.

\section{Wirkung von Benzoesäure und Oxybenzoesäuren.}

Die Gallussäure ist als eine Benzoesäure zu betrachten, die drei Hydroxylgruppen enthält. Um die Bedeutung dieser Atomgruppen in der gerinnungsfördernden Wirkung der Gallussäure feststellen zu können, wurde die Wirkung der Benzoe-, Salicyl-, Protocatechu- und Chinasäure auf die Blutgerinnung mit der der Gallussãure quantitativ vergleichend untersucht. Jede Substanz wurde in Wasser gelöst und nach Neutralisierung mit Natriumcarbonat mit Wasser verdünnt, um eine 10\%ige Lösung herzustellen. Diese wurde dem Tier in einer Menge von $1 \mathrm{ccm}$ pro $\mathrm{kg}$ Körpergewicht intravenös sehr langsam einverleibt.

Wie man in der Abbildung sieht, verursachen alle geprüften Substanzen eine, wenn auch nicht starke, so doch deutliche Verkürzung der Gerinnungszeit des Blutes, die bei der Gerbsäure und Chinasäure stärker als bei den anderen ist. Diese Gerinnbarkeitsänderung scheint auf einer eigentümlichen Wirkung der Substanzen, aber nicht auf ihrer Salzwirkung zu beruhen, denn das Natriumchlorid bewirkt in der gleichen Menge keine nachweisbare Verkürzung des Gerinnungszeit, sondern sogar manchmal

Abb. 1. Relative Gerinnungszeit des Blutes von Kaninchen vor und nach der intravenösen Darreichung von Benzoesäure und Oxybenzoesäuren in einer Menge von $0,1 \mathrm{~g}$ pro kg Körpergewicht.

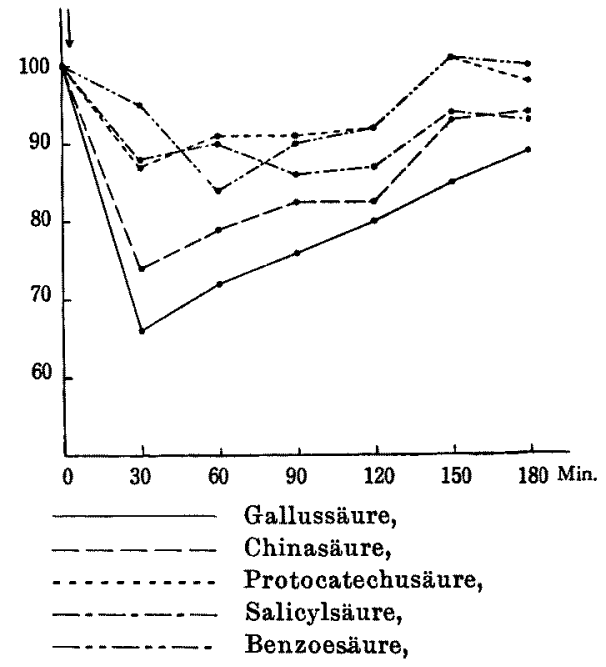


eine leichte Verlängerung. Dass die Benzoesäure, die in ihrem Moleküil keine Hydroxylgruppe hat, die Gerinnbarkeit steigert, wie die Oxybenzoesäuren, deutet vielleicht darauf hin, dass die Hydroxylgruppe im Molekül der Substanzen für ihre gerinnungsfördernde Wirkung nicht notwendig ist. Es lässt sich aber nicht leugnen, dass die Hydroxylgruppe an der Gerinnungsfördernden Wirkung im quantitativen Sinne beteiligt sein kann, da in der genannten Wirkung die Gallussäure der Salicylsäure und Protocatechusäure und diese widerum der Benzoesäure überlegen sind. Infolgedessen ist für die Entfaltung der gerinnungsfördernden Wirkung der Gerbsäure entweder der Benzolkern oder die Karboxylgruppe als der wesentliche Anteil zu betrachten. Um die Bedeutung der Karboxylgruppe klarstellen zu können, wurden ähnliche Versuche mit einigen Fettsäuren angestellt.

\section{Wirkung einiger Fettsäuren.}

Von den Fettsäuren wurden zuerst einbasische Säuren, nämlich Ameisen-, Essig-, Propion- und Buttersäure, ausgewählt, die auf die angegebene Weise in 10\%ige Lösungen übergeführt und für die Versuche gebraucht wurden. Die Versuchsergebnisse sind in Abb. 2 zusammengestellt.

Die Abbildung zeigt, dass die untersuchten einbasischen Säuren alle eine Verkürzung der Gerinnungszeit des Blutes bewirken, die an Intensität zwischen der der Gerbsäure und der Benzoesäure steht. Somit muss man verstehen, dass für die gerinnungsfördernde Wirkung dieser Substanzen, folglich auch der Gerbsäure, die Karboxylgruppe in ihrem Molekül der wesentliche Anteil ist.

Um die Beziehung zwischen der Zahl der Karboxylgruppen und der Intensität der gerinnungsfördernden

Abb. 2. Relative Gerinnungszeit des Blutes von Kaninchen vor and nach der intravenösen Darreichung einiger Fettsäuren.

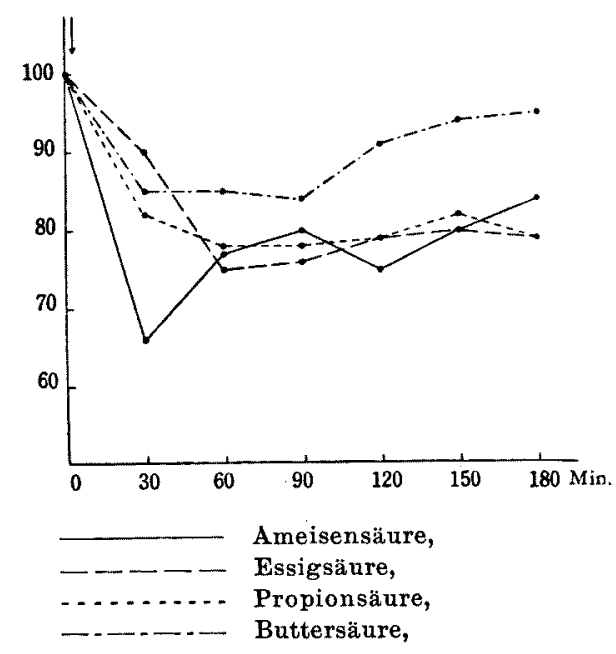


Wirkung klarzustellen, wurden die folgenden zweibasischen Säuren, die Oxal-, Malon- und Bernsteinsäure, untersucht.

Wie Abb. 3 zeigt, haben die letzteren zwei Säuren in einer Dosis von $0,1 \mathrm{~g}$ pro $\mathrm{kg}$ Körpergewicht keine oder nur eine leichte, schnell vorübergehende Verkürzung der Gerinnungszeit zur Folge. Bei der Oxalsäure starben die Tiere mehrere Minuten nach derDarreichung der angegebenen Dosis unter heftigen Konvulsionen, so dass die Versuche mit kleineren Dosen ausgeführt werden mussten. In einer Dosis von $0,05 \mathrm{~g}$ pro $\mathrm{kg}$ Körpergewicht, die die Tiere gut ertragen konnten, erfolgte im Gegenzatz zu den anderen Säuren eine leichte, doch unverkennbare Verlängerung in der Gerinnungszeit des Blutes, wie es allbekannt ist. Bei einer Dosis von 0,025 pro kg erfuhr die Gerinnungszeit dagegen eine deutliche Verkürzung, wie bereits von In $\mathrm{aba}^{3}$ ) bemerkt wurde. Die Verlängerung der Gerinnungszeit ist nach der allgemeinen Ansicht als Folge der Fällung von Kalziumionen im Blute zu betrachten, während die Verkürzung mit dieser in keinem Zusammenhang zu stehen scheint. Somit muss man verstehen, dass die Oxalsäure wie die anderen zweibasischen Säuren eine blutgerinnungsfördernde Wirkung hat, die aber unter Umständen durch ihre Eigenschaft, das Kalziumion zu fällen, überwunden wird.

Aus diesen Versuchen geht hervor, dass auch die zweibasischen Säuren eine blutgerinnungsfördernde Wirkung haben, die aber nicht stärker, sondern etwas schwächer als die einbasischen Säuren ist. Die Karboxylgruppe der genannten Substanzen scheint deshalb der wesentliche Anteil für die Entfaltung ihrer blutgerinnungsfördernde

3) In a b a, Okayama Igk. Z., 1935, 47, 3332.
Abb. 3. Relative Gerinnungszeit des Blutes ron Kaninchen vor und nach der intravenösen Darreichung einiger zweibasischen Säuren.

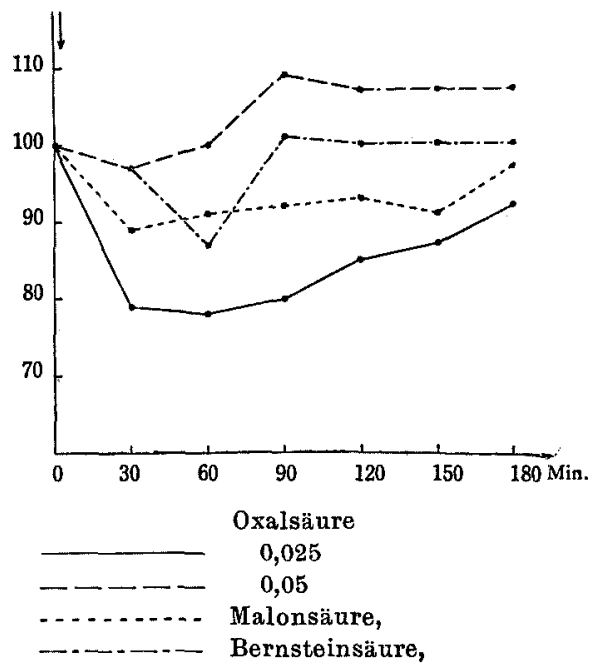


Wirkung zu sein, wenn sie auch mit der Wirkungsstärke in keinem Zusammenhang steht.

\section{Schluss.}

Die blutgerinnungsfördernde Wirkung der Gallussäure ist wahrscheinlich der Karboxylgruppe in ihrem Molekül zuzuschreiben, während die Wirkungsstärke von der Hydroxylgruppe in demselben beeinflusst zu werden scheint. 